\title{
Stroke in patients aged over 75 years: outcome and predictors
}

\author{
L. Kalra, D.H. Smith and P. Crome'
}

Department of Medicine for Elderly People, Bromley Hospitals, Bromley and ${ }^{1}$ Department of Health Care for Elderly People, King's College School of Medicine, London, UK

\begin{abstract}
Summary: The outcome and predictors of stroke rehabilitation were studied prospectively in 96 patients (mean age $81.3 \pm 5.4$ years) admitted to geriatric wards from a well-defined area over one year. Of these, $32(33 \%)$ died (median survival 11 days), $52(54 \%)$ returned home (median hospital stay 69 days) and $12(13 \%)$ required long-term care (median hospital stay 164 days). Deaths and discharges showed a bimodal pattern; nearly $\mathbf{4 0} \%$ of the patients died or were discharged within 2 weeks of admission. Early death correlated with level of consciousness $(P=0.02)$, neurological deficit $(P=0.01)$ and prestroke Barthel scores $(P=0.04)$ on admission. Patients with right- rather than left-sided hemiparesis $(P=0.02)$, good motor power $(P=0.002)$ and without sensory deficit/inattention $(P=0.002)$ were discharged early. Discharge home was adversely affected by poor awareness of deficit $(P=0.02)$, hemianopia $(P=0.03)$ and incontinence $(P=0.02)$ assessed at 2 weeks. Stroke survivors with Barthel score $<6$ and Mental Test Score $<4$ at 2 weeks after stroke required long-term care.
\end{abstract}

\section{Introduction}

An average district in England generates about 500 new strokes annually, of which $50-70 \%$ are hospitalized mainly for rehabilitation, nursing and social reasons. ${ }^{1,2}$ Approximately $41 \%$ of these patients are managed on geriatric wards and occupy nearly $21 \%$ of beds in the speciality. ${ }^{3,4}$ Treatment of stroke is expensive ${ }^{3}$ and accounts for nearly $10 \%$ of the district bed-day costs. ${ }^{5}$ These costs will escalate in future, because of the expected rise in the number of elderly stroke patients. ${ }^{5} \mathrm{~A}$ careful evaluation of the costs and benefits of various treatment options is essential before designing effective stroke rehabilitation services in the present climate of limited health-care resources.

The results of studies in stroke rehabilitation undertaken in the past are conflicting and are limited to relatively young (mean age under 75 years) stroke subjects. ${ }^{6-10}$ Treatment of stroke on general wards has been criticized, ${ }^{11}$ but the benefits of specialist units remain equivocal. ${ }^{8,10,12}$ Few studies have been undertaken in elderly subjects, many of whom have additional disability and, hence, poorer prognosis. ${ }^{13}$ There are no reports on stroke management on geriatric wards and the factors which influence outcome. The present study

Correspondence: L. Kalra, Ph.D., M.R.C.P. (UK), Department of Medicine for Elderly People, Orpington Hospital, Sevenoaks Road, Orpington BR6 9JU, UK. Accepted: 16 June 1992 is, to our knowledge, one of the first to audit stroke rehabilitation on geriatric wards. It provides baseline data with which future stroke management strategies in this age group can be compared and suggests clinical discriminants which will be helpful in evolving more effective rehabilitation protocols.

\section{Methods}

This one year prospective study was conducted in stroke patients over 75 years of age from a well-defined geographical area (estimated population $>75$ years $=7,400)^{14}$ admitted to a unit operating a 24 hour, age-related, acute admissions policy. The patch included the mainly suburban areas of Penge, Anerley, Beckenham, West Wickham and Hayes, all of which have an above average elderly population. The Standardized Mortality Ratio in this patch was 95.6 , and $5-7 \%$ of elderly population were below the Jarman Eight Index. ${ }^{14}$ During the one year period of the study, $97 \%$ of all patients over 75 years of age requiring hospital treatment were admitted to the unit. ${ }^{15}$

Stroke was defined as acute onset of neurological deficit lasting more than 24 hours or leading to death, with no apparent cause other than cerebrovascular disease. The diagnosis of stroke was based on history and clinical examination, but computed tomographic (CT) scanning was undertaken when 
the diagnosis was in doubt. Subjects with cerebral pathology other than cerebrovascular disease were excluded.

Details of age, gender, social circumstances, prestroke Barthel scores ${ }^{16}$ previous strokes, other medical problems and dementia were recorded. A detailed assessment within 72 hours of admission included level of consciousness (motor subscale of the Glasgow Coma Scale), ${ }^{17}$ side of stroke, subjective awareness of deficit (determined clinically by verbal or non-verbal response to the question 'Is there something wrong with the right/left side of your body?'), power in the arm and leg on the affected side (Medical Research Council grading), incoordination, hemianopia, dysphasia, dysphagia, sensory deficits, inattention (visual/sensory), continence, Hodkinson's abbreviated mental test score (MTS), ${ }^{18}$ mobility, ${ }^{19}$ and Barthel ADL scores. This assessment was repeated at weekly intervals.

The end points of the study were defined as: death, discharge home, placement in institutional care or hospital stay exceeding 24 weeks.

Stroke management was undertaken on mixed wards with stroke and non-stroke patients. Therapist cover included one full-time physiotherapist and one part-time occupational therapist (supported by one assistant each) per two wards of 20 beds. In addition, there was regular input from speech therapists, social workers and the patient placement officer.

Descriptive statistics have been used in data presentation and multiple regression analysis (stepwise deletion) was used to determine the effects of assessment variables on eventual outcome. The regression coefficient $(B)$ and the probability value $(P)$ of significant independent variables influencing outcome have been described where appropriate.

\section{Results}

Stroke was diagnosed in 102 patients and accounted for $9.9 \%(105 / 1,056)$ of the total admissions from the defined population representing a third of the district. Six patients were excluded because of non-cerebrovascular disease on CT scans.

\section{Demographic data}

The mean age of the 96 patients $(\mathrm{M}: \mathrm{F}$ ratio $=$ 28:72) included in the study was $81.3 \pm 5.4$ years. Previous strokes were seen in $18(18.8 \%)$ patients, $13(72 \%)$ on the same side and five $(28 \%)$ on the opposite side of the present stroke. The majority of patients $(n=74)$ were independent prior to stroke and lived alone $(n=58)$. Fifteen patients (living alone $=9$ ) required assistance for mobility and self care (mean Barthel score 11.9 \pm 2.2 ) and seven patients were admitted from long-term institutions (mean Barthel score 8.1 \pm 3.6). Dementia, diagnosed prior to the present admission, was present in $22(23 \%)$ patients, five of whom were admitted from long-term care.

\section{Initial assessment}

The consciousness level of patients ranged from $1-6$ (mean $4.2 \pm 2.6$ ) at the time of admission. Ten patients remained deeply comatose or died within 72 hours of admission and could not be assessed. In the remaining 86 patients, left hemiplegia was seen in $41(47.6 \%)$, right hemiplegia in $38(44.2 \%)$ and brainstem/cerebellar signs in seven $(8.2 \%)$ patients. The mean power in the affected arm was $2.4 \pm 1.5$ and $3.1 \pm 1.8$ in the affected leg. Sensory loss was present in $11(12.5 \%)$ patients, hemianopia in 34 $(39.5 \%)$, dysphasia in $17(44.7 \%$ of right hemiplegia), dysphagia in $17(19.7 \%)$, and sensory/ visual inattention in $46(53.4 \%)$ patients. Incontinence was seen in $68(79 \%)$ patients and 32 patients ( 28 with left hemiparesis) were subjectively unaware of the neurological deficit. The mean Barthel score at the time of admission was $5.2 \pm 6.7$ in the 86 patients assessed.

\section{Mortality}

Thirty-two patients (33\%) died during the study (median survival 11 days). The majority of the deaths were within the first 2 weeks of admission, with eight deaths within 72 hours, 15 within 7 days and 23 within 14 days of admission. Early death correlated with the level of consciousness $(B=$ $-0.114, P=0.021)$, severity of neurological deficit $(B=-0.058, P=0.008)$, dysphagia $(B=0.005$, $P=0.044)$ and pre-stroke Barthel scores $(B=0.0136, P=0.037)$. Age, gender, previous strokes, other medical problems or cognitive impairment prior to admission did not influence early mortality.

Nine patients died during rehabilitation, their median survival being 72 days. The cause of death was a further stroke in five patients (same side $=4$; opposite side $=1$ ), chest infection in three patients and an acute myocardial infarct in one patient.

\section{Rehabilitation outcome}

Of the 64 survivors, $52(80 \%)$ patients were discharged home (median length of hospital stay 69 days). Seventeen patients $(32.7 \%)$ were discharged within 3 weeks of admission. A complete resolution of neurological deficit was seen in nine patients. Neurological deficit improved considerably in other subjects, with improvements in power (predischarge mean $4.2 \pm 0.8$ ), mobility (predischarge mean $4.6 \pm 0.8$ ) and functional ability 
(predischarge mean Barthel $17.7 \pm 2.3$ ). These patients were able to cope with no or little additional help (shopping, cleaning) from the family or services. Factors that influenced early discharge included right- rather than left-sided stroke $(B=0.04, P=0.016)$, good motor power $(B=$ $0.0103, P=0.002$ ), consciousness level on admis$\operatorname{sion}(B=0.052, P=0.047)$, and absence of sensory deficit/inattention $(B=-0.034, P=0.0016)$ or incontinence $(B=-0.026, \quad P=0.008)$ during initial assessment. Early recovery was not influenced by age, gender, previous strokes or prestroke Barthel scores.

The median duration of stay of the 35 other patients eventually discharged home was 92 days. Twenty-three $(66 \%)$ were independently mobile with or without aids (mobility level 4-5), eight $(23 \%)$ were wheelchair independent and four patients required help with mobility and transfers (mobility level 2-3). The mean Barthel score at the time of discharge was $14.3 \pm 2.4$. All patients required additional social services support following discharge, a high input (personal care/home care) being required in $14(40 \%)$ patients. Ten $(29 \%)$ patients required further outpatient rehabilitation following discharge.

Twelve patients were transferred to nursing or residential homes (median duration of hospital stay 164 days). Of these, three had been discharged home previously with adequate support following a predischarge assessment at home. The functional ability of these patients was unchanged but longterm care was necessary because of social pressure.

In patients requiring longer rehabilitation assessment of physical or functional abilities (for example, age, gender, side of stroke, previous strokes, functional status prior to admission, other medical illness, severity of neurological deficit, consciousness levels, mental test scores, mobility and Barthel ADL scores) at the time of admission did not correlate with eventual discharge to home. A further assessment at 2 weeks was more predictive, showing an increased probability of requiring long-term care in patients with low MTS scores $(B=0.016, P=0.032)$, poor awareness of deficit $(B=0.019, \quad P=0.021)$, hemianopia/inattention $(B=-0.002, P=0.03)$, sensory deficit/inattention $(B=-0.007, P=0.008)$ and incontinence $(B=-0.031, P=0.019)$. Stroke survivors with Barthel score $<6$ and MTS $<4$ at 2 weeks failed to improve significantly and required long-term nursing care.

\section{Discussion}

The results show that $33 \%$ of hospitalized elderly stroke patients died, $54 \%$ returned home and $13 \%$ were transferred to institutional care. The number of patients returning home was higher than reported on general wards and comparable to that on stroke rehabilitation units. , $6,8,9,20$

Several factors may have contributed to this observation. Poor outcome on general wards has been attributed to misunderstanding and rivalries between professionals, breakdown of communication and ill-prepared or unplanned discharges. ${ }^{11}$ These problems are less likely to occur on geriatric wards, where a multidisciplinary team approach to patient care, consultation with carers and planned discharges are normal practice. Not surprisingly, recent studies have failed to show any difference in outcome between specialist units and geriatric wards. $^{12,20}$ It may also have been possible to discharge patients with a high level of disability, firstly because of lower expectations of well-being amongst elderly people and, secondly, because of the community support systems for elderly people developed during the last two decades. Finally, it is possible that therapy resources and skills may have been diverted from general wards to stroke rehabilitation units during previous studies, with adverse effects on staff motivation and rehabilitation potential, resulting in poorer outcome.

The median length of hospital stay for survivors in the present study (69 days) was greater than that of 29-55 days reported elsewhere. ${ }^{4,9,20}$ It is possible that age (mean 81 years in this study compared with $<75$ years in other studies), other medical or locomotor problems and sensory or cognitive impairment may have contributed to slower recovery. On the other hand, a longer hospital stay may have been the result of poorly coordinated and improperly targeted therapy. ${ }^{21}$ There is some evidence that patients managed on special units recover more quickly and there may be savings on hospital bed occupancy. ${ }^{5,20}$ Hence, the benefit of a stroke rehabilitation unit for elderly people may lie in reducing hospital stay rather than improving outcome.

The success of a stroke rehabilitation unit would depend upon the effective utilization of its potential, mainly by appropriate patient selection. ${ }^{22}$ Specialist units would be of little advantage in patients who would do well or those who would do badly whatever the therapy input. However, the early and reliable identification of such patients and those most likely to benefit from intensive rehabilitation has presented problems. ${ }^{5,23}$ The timing of transfer to stroke rehabilitation units may be another important determinant.

Nearly $40 \%$ of the patients were discharged or died within 2 weeks of admission to this study. Also, clinical assessment at 2 weeks correlated significantly with eventual discharge home or the need for long-term care. Hence, this would appear to be the appropriate time to assess patients for specialist rehabilitation. 
Stroke survivors with low Barthel and MTS score in the absence of an acute illness 2 weeks after admission appear to have limited rehabilitation potential and alternative management strategies need to be considered. The prospects of discharge home (with or without support) are also poor in patients who continue to have severe motor deficit, sensory/perceptual problems, hemianopia and incontinence 2 weeks after admission.

The clinical determinants which significantly influenced the outcome of stroke rehabilitation of elderly patients in this study are in general agreement with those seen in other studies. ${ }^{24-27}$ Prognostic scores based on clinical determinants have been suggested for younger stroke patients. ${ }^{24}$ The similarity in clinical determinants of stroke rehabilitation outcome in the elderly suggests that is may be possible to modify these scores for use in the elderly to help target scarce therapy resources to patients most likely to benefit from intensive input.

\section{References}

1. Wade, D.T. \& Langton Hewer, R. Hospital admission for acute stroke: who, for how long and to what effect? $J$ Epidemiol Comm Health 1985, 39: 347-352.

2. Bamford, J., Sandercock, P., Warlow, C. \& Gray, M. Why are patients with acute stroke admitted to hospital? $\mathrm{Br}$ Med $\mathrm{J}$ 1986, 1: $1369-1372$.

3. Office of Health Economics. Stroke. Office of Health Economics, London, 1988.

4. Wade, D.T., Wood, V.A. \& Langton Hewer, R. Use of hospital resources by acute stroke patients. $J R$ Coll Phys 1985, 19: 48-52.

5. Langton Hewer, R. Rehabilitation after stroke. $Q J$ Med 1990, 279: 659-674.

6. Hamrin, E. Early activation in stroke: does it make a difference? Scand J Rehabil Med 1982, 14: 101-109.

7. Hamrin, E. One year after stroke: a followup of an experimental study. Scand J Rehabil Med 1982, 14: 111-116.

8. Stevens, R.S., Ambler, N.R. \& Warren, M.D. A randomised controlled trial of a stroke rehabilitation ward. Age Ageing 1984, 13: 65-75.

9. Garraway, W.M., Akhtar, A.J., Prescott, R.J. \& Hockey, L. Management of acute stroke in the elderly: Preliminary results of a controlled trial. Br Med J 1980, 280: 1040-1043.

10. Garraway, W.M., Akhtar, A.J., Hockey, L. \& Prescott, R.J. Management of acute stroke in the elderly: follow-up of a controlled trial. Br Med J 1980, 281: 827-829.

11. King's Fund Consensus Statement. The Treatment of Stroke. King's Fund, London, 1988.

12. Edmans, J.A. \& Towle, D. Comparison of stroke unit and non-stroke unit in patients on independence in ADL. $B r J$ Occup Therapy 1990, 53: 415-418.

13. Carstairs, V. Resource consumption and cost to the community. In: Gillingham, F.J., Mawdsley, C. \& Williams, A.E. (eds) Stroke. Churchill Livingstone, New York, 1976, pp. 516-528.

14. NHS Health Advisory Service. Report on Services for Mentally IIl and Elderly People in the Bromley Health District, 1990.

15. Bromley Health Authority Department of Public Health Medicine. Report on the Health of Bromley's Population, 1990.

16. Wade, D.T \& Collin, C. The Barthel ADL index: a standard measure of physical disability? Int Disability Stud 1988, 10: 64-67.

17. Wade, D.T. Assessing disability after acute stroke. In: Clifford Rose, F. (ed.) Stroke: Epidemiological, Therapeutic and Socioeconomic Aspects. Royal Society of Medicine, London, 1986, pp. 101-114.

18. Qureshi, K.N. \& Hodkinson, H.M. Evaluation of a tenquestion mental test in the institutionalised elderly. Age Ageing 1974, 3: 152-157.

19. Holden, M.K., Gill, K.M., Magliozzi, M.R., Nathan, J. \& Piehl-Baker, L. Clinical gait assessment in the neurologically impaired: reliability and meaningfulness. Phys Ther 1984, 64:O 35-40.

20. Friedman, P.J. Stroke rehabilitation in the elderly: a neweق patient management system. NZ Med J 1990, 103: 234-236.

21. Wade, D.T., Skilbeck, C.E., Langton Hewer, R. \& Wood, V.A. Therapy after stroke: amounts, determinants and effects. Int Rehabil Med 1984, 6: 105-110.

22. Young, A. Assessment for rehabilitation after stroke. Ann Acad Med Singapore 1988, 17: 267-274.

23. Osberg, J.S., Haley, S.M., McGinnis, G.E. \& DeJong, G. Characteristics of cost outliers who did not benefit from stroke rehabilitation. Am J Phys Med Rehabil 1990, 69: 117-125.

24. Prescott, R.J., Garraway, W.M. \& Akhtar, A.J. Predicting functional outcome following acute stroke using a standard clinical examination. Stroke 1982, 13: 641-647.

25. Allen, C.M.C. Predicting recovery after acute stroke. $\mathrm{Br} J$ Hosp Med 1984, 31: 428-434.

26. Anderson, T.P. Studies up to 1980 on stroke rehabilitation outcomes. Stroke 1990, 21: 1143-1145.

27. Shah, S., Vanclay, F. \& Cooper, B. Stroke rehabilitation: Australian patient profile and functional outcome. J Clin Epidemiol 1991, 44: 21-28. 